\title{
Binding of Cryptococcus neoformans by Human Cultured Macrophages Requirements for Multiple Complement Receptors and Actin
}

\author{
Stuart M. Levitz and Abdulmoneim Tabuni \\ Evans Memorial Department of Clinical Research and the Department of Medicine, The University Hospital, \\ Boston University Medical Center, Boston, Massachusetts 02118
}

\begin{abstract}
We studied the receptors on human cultured macrophages (MO-M $\phi)$ responsible for binding encapsulated and isogenic mutant acapsular strains of Cryptococcus neoformans, and whether such binding leads to a phagocytic event. Both strains required opsonization with complement components in normal human serum in order for binding to occur. Binding of the acapsular, but not the encapsulated, strain led to phagocytosis. MAb directed against any of the three defined complement receptors (CR) on MO-M $\phi(C R 1, C R 3$, and CR4) profoundly inhibited binding of serum-opsonized encapsulated (and to a lesser extent acapsular) organisms to MO-M $\phi$. Immunofluorescence studies demonstrated migration of $\mathrm{CR}$ to the area of the cryptococcal binding site. Trypsin and elastase inhibited binding of encapsulated and, to a lesser extent, acapsular yeasts to MO-M $\phi$. Binding of encapsulated $C$. neoformans was profoundly inhibited by incubation in the cold or by inhibitors of receptor capping and actin microfilaments. Thus, multiple $\mathbf{C R}$ appear to contribute to binding of serum-opsonized encapsulated $C$. neoformans by MO-M $\phi$. Binding is an energy-dependent process that requires conformational changes in actin yet does not lead to phagocytosis of the organism. In contrast, energy is not required for binding of acapsular yeasts by MO-M $\phi$ and binding triggers phagocytosis. (J. Clin. Invest. 1991. 87:528-535.) Key words: cryptococcosis • divalent cations • fungus $\bullet$ phagocytosis $\bullet$ receptor capping
\end{abstract}

\section{Introduction}

Infections due to the encapsulated yeastlike fungus Cryptococcus neoformans are significant causes of morbidity and mortality in patients with impaired cell-mediated immunity, especially those with AIDS (1). Macrophages are thought to play a major role in host defenses against this opportunistic pathogen (2-6). In the absence of specific antibody, a functional complement system has been shown to be required for binding of $C$. neoformans to human macrophages in vitro $(7,8)$. C. neoformans is a potent activator of the complement cascade, with large amounts of $\mathrm{C} 3$, primarily in the form of $\mathrm{iC} 3 \mathrm{~b}$, deposited on the surface of the capsule after incubation in normal human serum (8-11). Binding by phagocytes of $C$. neoformans opsonized with human serum occurs at a relatively low rate com-

Address correspondence to Stuart M. Levitz, M.D., Room E540, The University Hospital, 88 E. Newton St., Boston, MA 02118.

Received for publication 21 February 1990 and in revised form 10 August 1990.

J. Clin. Invest.

(C) The American Society for Clinical Investigation, Inc.

$0021-9738 / 91 / 02 / 0528 / 08 \$ 2.00$

Volume 87, February 1991, 528-535 pared with mutant yeasts lacking capsule, despite being covered with seemingly sufficient amounts of $\mathrm{iC} 3 \mathrm{~b}(5,6,12)$. Whereas capsule confers upon $C$. neoformans a negative surface charge and a hydrophilic surface, the antibinding effects of capsule can be dissociated from these physicochemical properties $(13,14)$.

Human monocyte-derived macrophages (MO-M $\phi)^{1}$ and other phagocytes have been shown to possess at least three distinct complement receptors (CR) that recognize $\mathrm{C} 3$ degradation fragments (reviewed in reference 15). CR1 (CD35) and CR3 (CD1 lb/CD18) have greatest affinity for particles coated with $\mathrm{C} 3 \mathrm{~b}$ and $\mathrm{iC} 3 \mathrm{~b}$, respectively, whereas CR4 (CD11c/CD18, p150,95) preferentially recognizes both iC3b-and C3dg-coated stimuli. Although CR 3 and CR4 share a common $\beta$ chain (CD18), their complement-binding activities have been localized to regions on their distinct $\alpha$ chains $(15,16)$. These receptors can be differentiated on the basis of their reactivities to $\mathrm{MAb}$, their sensitivities to proteases and their requirements for temperature and divalent cations (17-20). In addition to sites for binding complement, the $\alpha$ chain of CR3 also appears to have sites for binding $\beta$-glucans (21), whereas the common $\beta$ chain of CR3, CR4, and LFA-1 (the third member of the CD18 family) has been shown to play a role in cellular adhesion and in binding unopsonized Histoplasma capsulatum and bacterial endotoxin $(18,22,23)$.

Whether a microorganism is able to establish itself as a pathogen depends to a large extent upon which receptors on host cells bind to it, and the consequences of that binding. Therefore, in this paper, we investigated the requirements for binding (attachment) of serum-opsonized encapsulated and mutant acapsular strains of $C$. neoformans to MO-M $\phi$, and whether binding leads to actual phagocytosis (internalization). Taken together, several lines of evidence suggested that multiple CR are involved in binding of serum-opsonized encapsulated $C$. neoformans by MO-M $\phi$. Moreover, binding was an energy-dependent process that required conformational changes in actin yet did not lead to phagocytosis of the organism. In contrast, binding of serum-opsonized acapsular $C$. neoformans by MO-M $\phi$ did not require energy and led to phagocytosis of the yeast.

\section{Methods}

Materials. All reagents used, except where noted otherwise, were obtained in the highest quality available from Sigma Chemical Co. (St. Louis, MO). Medium used in all experiments, unless stated otherwise, was RPMI 1640 (Gibco, Grand Island, NY) supplemented with L-glutamine, penicillin, and streptomycin. Human neutrophil elastase was obtained from Elastin Products Corp. (Pacific, MO). Sheep erythro-

1. Abbreviations used in this paper: $\mathrm{CR}$, complement receptor(s); EC3b and $\mathrm{EiC} 3 \mathrm{~b}$, erythrocytes coated with $\mathrm{C} 3 \mathrm{~b}$ and $\mathrm{iC} 3 \mathrm{~b}$; MO-M $\phi$, monocyte-derived macrophages; PHS, pooled human serum. 
cytes selectively opsonized with $\mathrm{C} 3 \mathrm{~b}$ (EC3b) and iC3b (EiC3b) were prepared as described previously using purified components of the alternative complement pathway (24) and were a generous gift of Dr. Simon Newman (Cincinnati, $\mathrm{OH}$ ).

Opsonins. Pooled human serum (PHS) was obtained by pooling serum from 10 healthy volunteers and was kept in aliquots at $-70^{\circ} \mathrm{C}$ until use. PHS was heat-inactivated by incubation at $56^{\circ} \mathrm{C}$ for $30 \mathrm{~min}$. Two separate techniques were used to deplete PHS of antibodies reactive with $C$. neoformans. First, antibodies were adsorbed to the yeasts at $4^{\circ} \mathrm{C}$ in the presence of EDTA, as previously described (25). Second, PHS was incubated three times with Protein A Sepharose 4B (Pharmacia Fine Chemicals; Piscataway, NJ) at $0^{\circ} \mathrm{C}$ in the presence of EDTA. PHS was dialyzed against PBS, concentrated to its original volume with an Amicon filter and recalcified. This second technique depleted over $97 \%$ of the IgG in the PHS (from 12.1 to $0.3 \mathrm{mg} / \mathrm{ml}$ ) as measured by nephelometry. Anticapsular antibody, a generous gift of Dr. John Bennett (Bethesda, MD), was prepared by immunizing rabbits with serotype $D$ capsular polysaccharide, and used at a subagglutinating concentration (1:8,600 final dilution).

Monoclonal antibodies. Mouse MAb directed against human macrophage surface antigens were as follows: anti-CR1 $\left(\operatorname{lgG}_{1}\right.$, binds to CR1, Becton-Dickinson, Mountain View, CA); OKM10 and OKM ( $\mathrm{IgG}_{2 \mathrm{a}}$ and $\mathrm{IgG}_{2 \mathrm{~b}}$, respectively, both bind to the $\alpha$ chain of CR3, kindly provided by Dr. Patricia Rao, Ortho Pharmaceutical Corp. Raritan, NJ) (26); anti-leu-M5 ( $\mathrm{IgG}_{2 b}$, binds to the $\alpha$ chain of CR4, BectonDickinson); $7 \mathrm{C} 3$ ( $\operatorname{lgG}_{3}$, binds to sites on neutrophils, but not MO-M $\phi$, kindly provided by Dr. David Melnick, Bethesda, MD) (27); and IV.3 ( $\operatorname{lgG}_{2 b}$, binds to FcRII, kindly provided by Dr. Michael Fanger, Hanover, $\mathrm{NH}$ ) (28). Commercially obtained MAb were dialyzed twice against $500 \mathrm{vol}$ of PBS to remove azide. MAb were used at a final concentration of $25 \mu \mathrm{g} / \mathrm{ml}$, except for anti-CR 1 and anti-leu-M5 which were used at $12.5 \mu \mathrm{g} / \mathrm{ml}$ and $4 \mu \mathrm{g} / \mathrm{ml}$, respectively. $\mathrm{F}(\mathrm{ab})$ fragments of OKM10 were prepared using immobilized papain, according to the manufacturer's directions (Pierce Chemical Co., Rockford, IL). Residual $\mathrm{Fc}$ fragments and any undigested MAb were removed with Protein A-Sepharose. Purity of the preparation was ascertained by SDS-PAGE.

Human MO-M $\phi$. Heparinized blood was obtained by venipuncture from normal volunteers and the PBMC purified by centrifugation over a cushion of Ficoll-Hypaque. Cells were suspended at $5 \times 10^{5} / \mathrm{ml}$ in medium containing $10 \%$ human $\mathrm{AB}$ male serum and $100 \mu \mathrm{l}$ added per well of 96-well flat-bottom polystyrene tissue culture plates (No. 25860, Corning Glass Works, Corning, NY). For some experiments with monoclonal antibodies, to conserve reagents, "half area" 96-well plates were used (Costar, Cambridge, MA). Plates were incubated in a $37^{\circ} \mathrm{C}$, $5 \% \mathrm{CO}_{2}$ humidified environment, washed after $24 \mathrm{~h}$ to remove nonadherent cells and cultured for 6-21 d before challenge with $C$. neoformans. Variation of the culture period over this range did not affect results. After the washing step, the remaining cells in the well were $>98 \%$ monocytes as assessed by morphology and nonspecific esterase staining. For immunofluorescent studies determining the localization of receptors, as well as the assay to distinguish attached from phagocytosed organisms, MO-M $\phi$ were cultured as above, except in eightchamber glass slides (Lab-Tek, Miles Scientific, Naperville, IL).

C. neoformans. Encapsulated, serotype D strain B3501 (also known as strain MP415) and acapsular, isogenic mutant strain CAP67 were maintained and harvested as previously described (6). To facilitate rapid, accurate identification of yeasts, most experiments used FITCor RITC-labeled, heat-killed organisms. Selected experiments yielded similar results with live, unlabeled organisms. Yeasts were heat-killed by immersion in $a 0^{\circ} \mathrm{C}$ water bath for $30 \mathrm{~min}$ and then labeled with either FITC or RITC as previously described $(5,6)$. C. neoformans were preopsonized by incubation in either PHS or anticapsular antibody for $30 \mathrm{~min}$ at $37^{\circ} \mathrm{C}$, followed by three washes in PBS.

Binding assays. Wells containing MO-M $\phi$ were washed with medium, except for experiments examining divalent cation requirements where cells were washed with PBS containing glucose and the indicated cations. Monolayers were then treated with MAb, proteases or inhibitors, and preopsonized $C$. neoformans $\left(2.5 \times 10^{5}\right.$ and $2.5 \times 10^{4}$ per well for the encapsulated and acapsular strains, respectively) was added. The 10-fold higher inoculum of the encapsulated strain was necessary to correct for the less efficient MO-M $\phi$ binding of encapsulated, compared with acapsular, organisms. For the protease experiments, proteases were washed off before addition of yeasts so that complement and other components on the surface of the organisms would not be cleaved. MO-M $\phi$ and yeasts were incubated at $37^{\circ} \mathrm{C}$ for 5 to $30 \mathrm{~min}$ following which unbound organisms were washed off and the monolayer fixed with $1 \%$ formaldehyde in PBS. Using an inverted microscope at 200 $\times, 100-200$ MO-M $\phi$ were randomly selected under brigh field and then examined under epifluorescence to determine the number of yeasts associated per MO-M $\phi$. Binding index represents the number of cell-associated yeasts per $100 \mathrm{MO}-\mathrm{M} \phi$. Due to variability in baseline binding indices between experiments, results are expressed as percent inhibition of binding which was calculated as: $[1$ - (binding index of experimental group/binding index of control group)] $\times 100$.

Assay to distinguished attached from fully internalized organisms. The above binding assay does not distinguish between organisms bound to the surface of MO-M $\phi$ versus those that have been fully phagocytosed (internalized). To make this distinction, our previously described Uvitex assay, with slight modifications, was used $(5,25)$. MO-M $\phi$ were cultured as above, except in wells of eight-chamber tissue culture slides (Lab-Tek, Miles Scientific) at $4 \times 10^{5}$ per well. After three washes with RPMI 1640, MO-M $\phi$ were challenged for $30 \mathrm{~min}$ at $37^{\circ} \mathrm{C}$ with RITC-labeled $C$. neoformans in the presence of $10 \%$ PHS. Wells were washed with PBS, and the cells incubated with $0.1 \%$ Uvitex (diaethanol or Fungiqual, Ciba-Giegy, Basel, Switzerland) for $1 \mathrm{~min}$ at $23^{\circ} \mathrm{C}$. Wells were again washed in PBS and the cells fixed with $1 \%$ formaldehyde. Using an epifluorescent microscope, 200 cell-associated yeasts per well were identified after excitation at $546 \mathrm{~nm}$, and then examined with UV excitation to determine which yeasts stained with Uvitex. Organisms that stain with Uvitex under these conditions are bound but not internalized, whereas those that do not stain are fully internalized $(5,25)$. Bound only organisms were also distinguished from those internalized by examination under phase-contrast microscopy, as described (29).

Immunofluorescence localization of complement receptors. Wells containing MO-M $\phi$ were sequentially incubated with $(a)$ unlabeled encapsulated or acapsular strains of $C$. neoformans for $10 \mathrm{~min}$; $(b)$

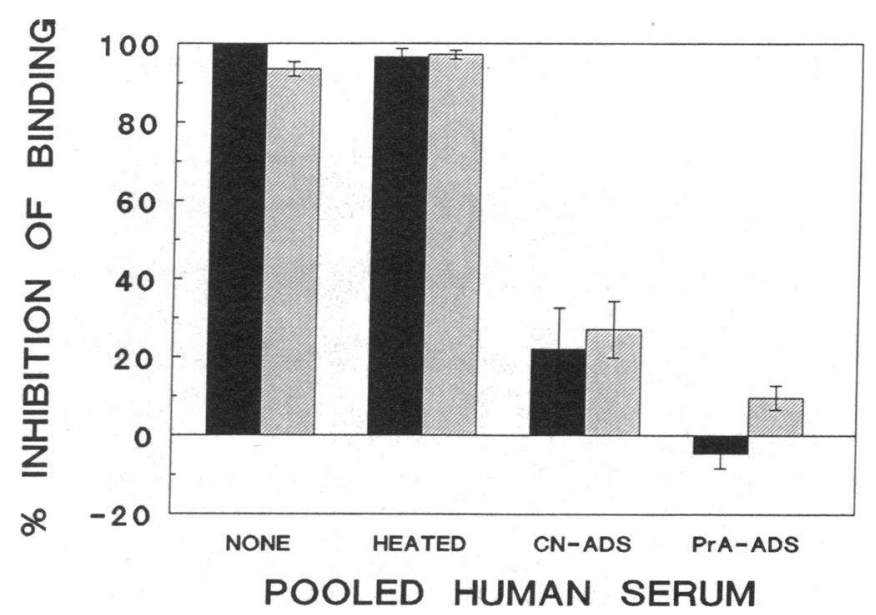

Figure 1. Role of opsonins in binding of $C$. neoformans to MO-M $\phi$. MO-M $\phi$ were incubated at $37^{\circ} \mathrm{C}$ for $30 \mathrm{~min}$ with encapsulated $(\square)$ or acapsular (四) strains of $C$. neoformans in the presence of $10 \%$ PHS, no PHS (NONE), heat-inactivated PHS (HEATED), or PHS depleted of reactive antibodies by adsorption with $C$. neoformans ( $C N-A D S)$ or protein $\mathrm{A}(\operatorname{PrA}-A D S)$. Results are expressed as percent inhibition of binding compared with values obtained with untreated PHS and represent means $\pm S E M$ of three triplicate experiments. 
Table I. Internalization of Selectively Opsonized Encapsulated and Acapsular C. neoformans by $M O-M \phi$

\begin{tabular}{llc}
\hline \multicolumn{1}{c}{ Strain } & \multicolumn{1}{c}{ Opsonins } & \% Internalized \\
\hline Encapsulated & PHS & $23 \pm 1$ \\
Encapsulated & Ig-depleted PHS & $19 \pm 2$ \\
Encapsulated & Anti-cap Ig & $64 \pm 6$ \\
Encapsulated & PHS + Anti-cap Ig & $75 \pm 5$ \\
Acapsular & PHS & $88 \pm 4$ \\
Acapsular & Ig-depleted PHS & $87 \pm 5$ \\
\hline
\end{tabular}

MO-M $\phi$ were incuabated with a selectively opsonized encapsulated or acapsular strain of $C$. neoformans for $30 \mathrm{~min}$ at $37^{\circ} \mathrm{C}$. Of the organisms bound to MO-M $\phi$, the percentage that was completely internalized (phagocytosed) was determined by the Uvitex assay. Rabbit anticapsular immunoglobulin (anti-cap Ig) was used at a subagglutinating concentration. Results represent the means \pm SEM of two to four separate duplicate experiments.
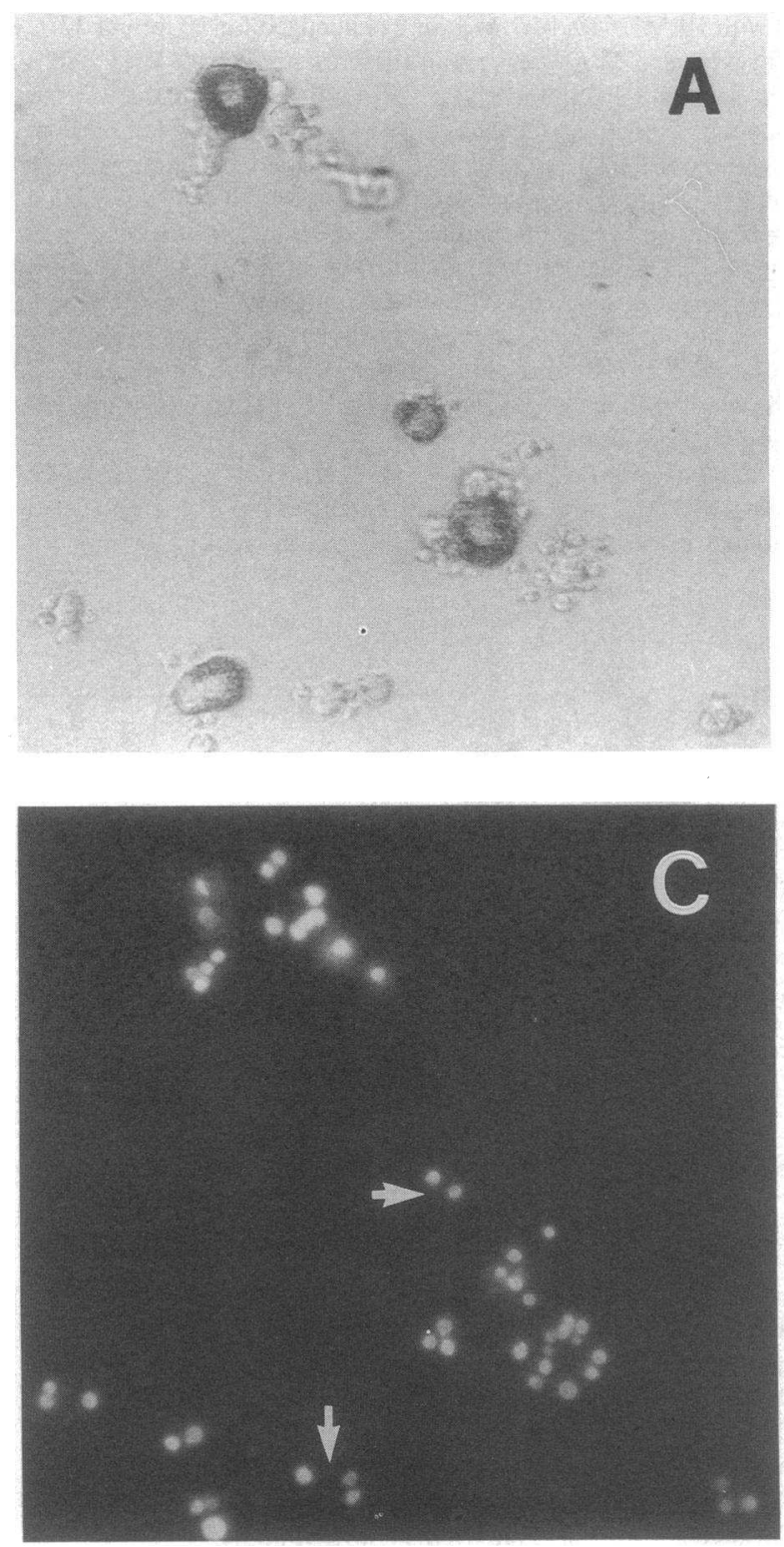

MAb (5 $\mu \mathrm{g} / \mathrm{ml})$ for $10 \mathrm{~min}$; (c) 1:2,000 dilution of Biotin-SP F(ab)' fragment goat anti-mouse IgG (Jackson Immunoresearch Laboratories, West Grove, PA) for $30 \mathrm{~min}$; (d) fluorescein-conjugated strepavidin $(50 \mu \mathrm{g} / \mathrm{ml}$; Jackson Immunoresearch Laboratories, West Grove, PA) for $30 \mathrm{~min}$; ard (e) $1 \%$ formaldehyde. Wells were washed six times with medium between the above incubations. MO-M $\phi$ containing $C$. neoformans were identified under light microscopy, and then observed under epifluorescence where the distribution of fluorescent staining was noted. For all experiments, controls were done omitting incubations $b, c$, or $d$. Under such conditions, fluorescence was not seen.

\section{Results}

Opsonic factors necessary for binding and internalization of Cryptococci by MO-M $\phi$. Initial experiments examined the opsonic requirements for binding of encapsulated and acapsular C. neoformans to human MO-M $\phi$ (Fig. 1). Omission of opsonins, or heat inactivation of PHS, resulted in nearly complete inhibition of binding compared with wells containing PHS.

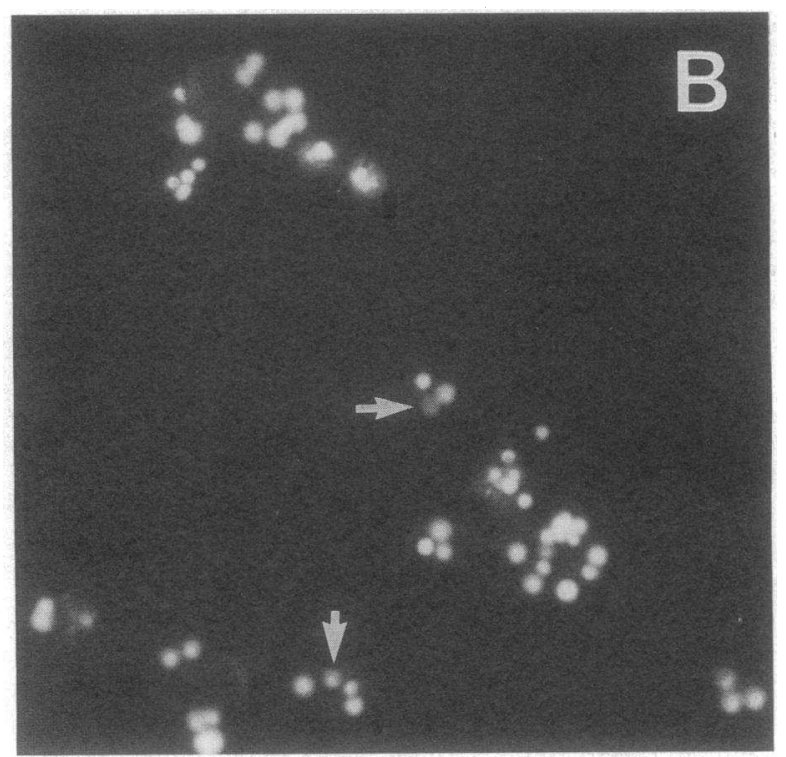

Figure 2. Internalization of encapsulated $C$. neoformans by MO-M $\phi$, as determined by the Uvitex assay. MO-M $\phi$ were incubated with RITC-labeled C. neoformans in the presence of $10 \%$ PHS for $30 \mathrm{~min}$ and then stained with Uvitex. Only extracellular yeasts stain with Uvitex. Brightfield photomicrograph $(A)$ demonstrates MO-M $\phi$, some of which contain cell-associated $C$. neoformans. Note that the yeast cells tend to form rosettes around the MO-M $\phi$. Fluorescent photomicrograph of the same field taken with peak excitation at 490 $\mathrm{nm}(B)$ and $545 \mathrm{~nm}(C)$ demonstrates $C$. neoformans with RITC and Uvitex staining, respectively. Most of the RITC-stained organisms also stain with Uvitex, indicating that they are extracellular. Arrows point to rare intracellular organisms that stain with RITC, but not Uvitex. 

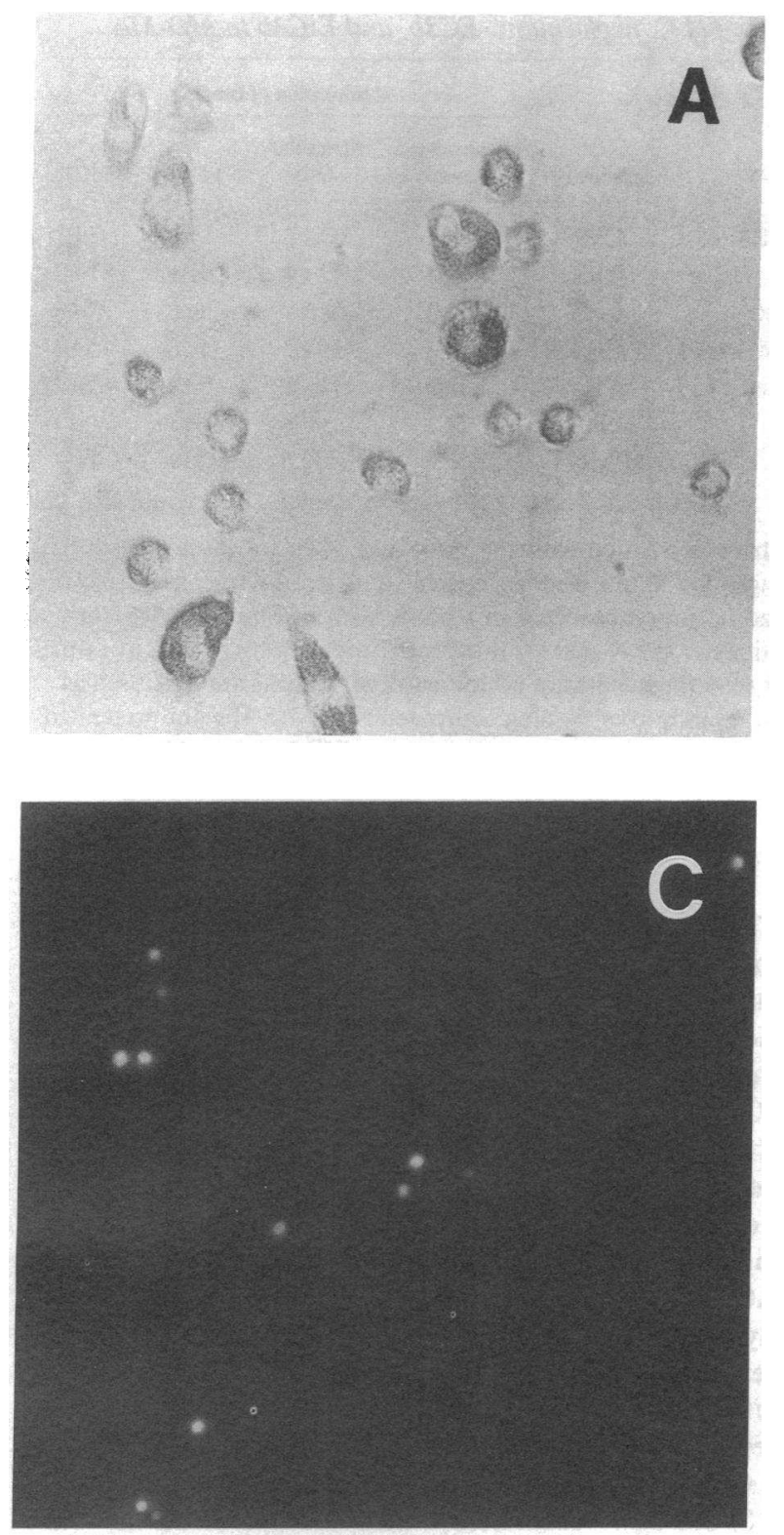

Depleting PHS of immunoglobulins reactive with $C$. neoformans by either of two methods had a minimal effect on binding. Thus, consistent with previous studies $(7,8)$, a heat-labile factor in human serum, presumably complement, appeared to be required for binding.

Using the Uvitex assay, we next examined whether the bound organisms were internalized (Table I, Figs. 2 and 3). Of the cell-associated serum-opsonized encapsulated organisms, only $23 \%$ were internalized by the MO-M $\phi$. Depleting PHS of immunoglobulins reactive with $C$. neoformans did not significantly alter the percentage of organisms internalized. However, when the yeasts were opsonized with specific anticapsular antibody, most of the bound organisms were internalized. In contrast, $88 \%$ of acapsular yeasts were completely internalized after opsonization in PHS alone, even if the PHS was depleted of immunoglobulins reactive with $C$. neoformans. Identical trends were observed using phase-contrast microscopy instead of the Uvitex assay (data not shown).

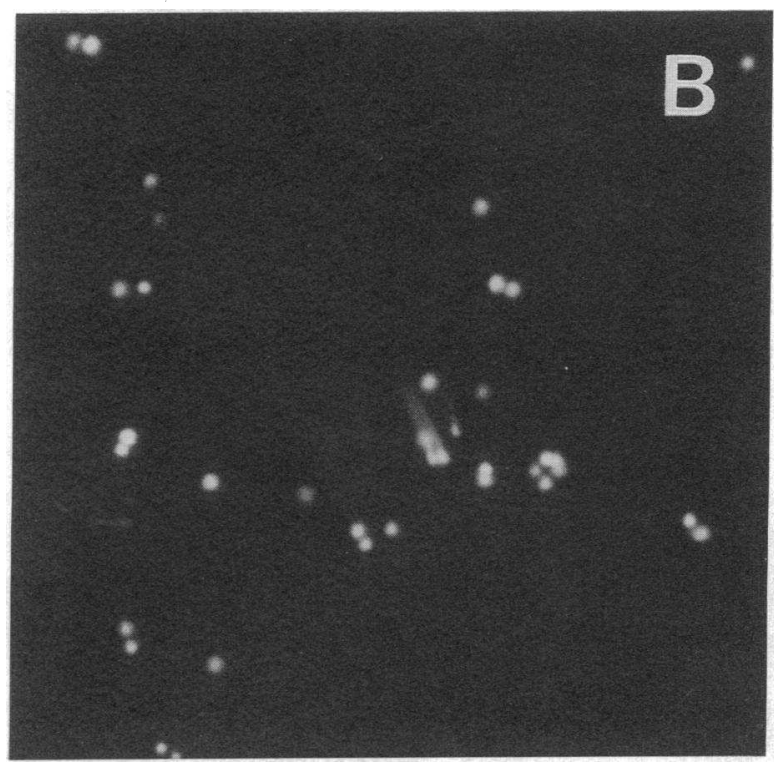

Figure 3. Internalization of acapsular $C$. neoformans by MO-M $\phi$, as determined by the Uvitex assay. Fig. 3, $A-C$, correspond to Fig. 2 , $A-C$, except acapsular $C$. neoformans were used. Note that rosettes of organisms are not seen on the brightfield photomicrograph. The majority of yeast cells do not stain with Uvitex, indicating that they are intracellular.

Inhibition of binding by anti-receptor MAb. A panel of MAb known to be reactive with specific $C R$ and FcR on phagocytes was examined for its ability to inhibit binding of PHS-opsonized encapsulated and acapsular $C$. neoformans to MO-M $\phi$ (Table II). To control for the specificity, EC3b and EiC3b were included as targets in some of the experiments. MAb directed against any of the three defined CR on MO-M $\phi$ (CR1, CR3, and CR4) profoundly inhibited binding of serum-opsonized encapsulated (and to a lesser extent acapsular) $C$. neoformans to $\mathrm{MO}-\mathrm{M} \phi . \mathrm{F}(\mathrm{ab})$ fragments of OKM10 also inhibited, although to a lesser extent than whole MAb. In contrast, MAb IV.3 directed against FcRII, or MAb 7C3 which reacts with PMN (but not MO-M $\phi$ ), did not inhibit. If encapsulated yeasts were opsonized with IgG in lieu of PHS, then MAb directed against CR no longer inhibited (data not shown). Binding of EC3b to MO-M $\phi$ was not inhibited by any of the MAb tested. Even anti-CR1 did not inhibit binding, suggesting that this MAb does not block the $\mathrm{C} 3 \mathrm{~b}$ binding site on CR 1 . Both of the 

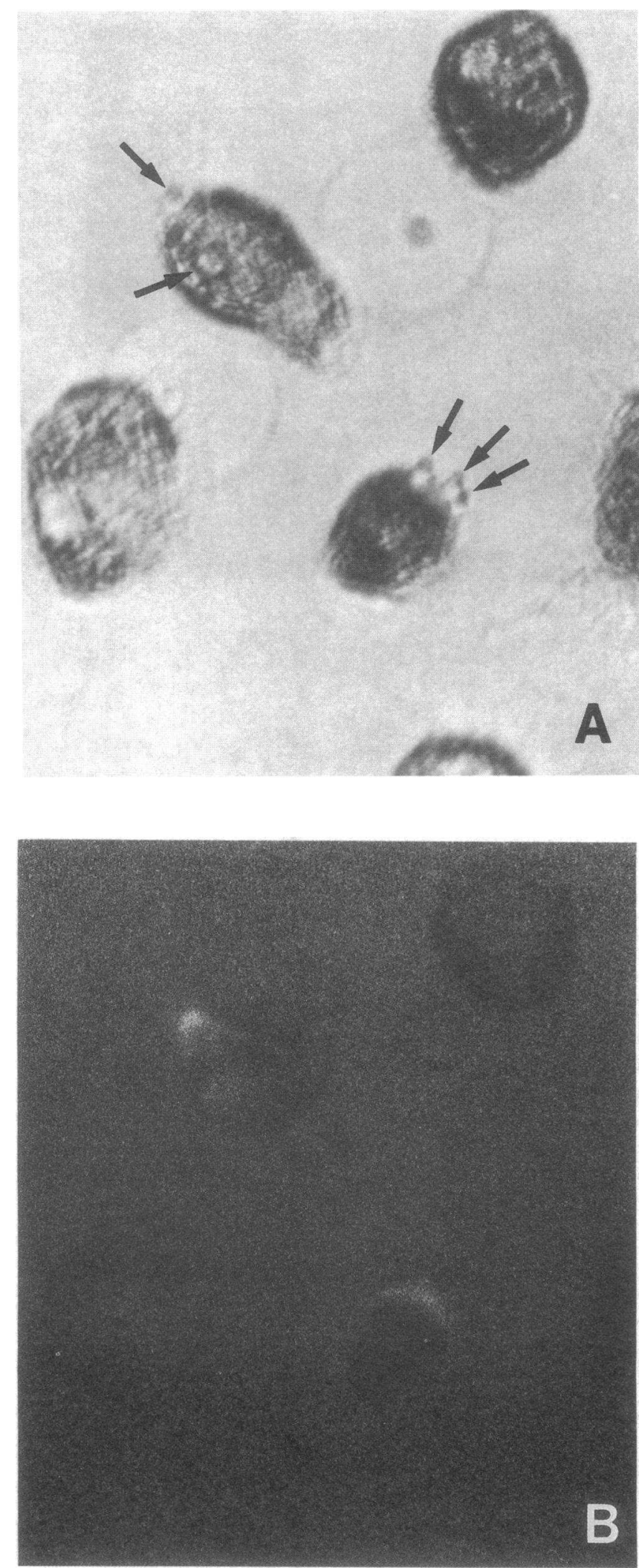

Figure 4. Brightfield $(A)$ and corresponding fluorescent $(B)$ photomicrographs of MO-M $\phi$ incubated with $C$. neoformans for 10 min followed by anti-leu-M5 (anti-CR4). Immunofluorescent localization of anti-leu-M5 was then accomplished by sequential incubations with biotin-conjugated $F(a b)_{2}^{\prime}$ fragment goat anti-mouse IgG and fluorescein-conjugated strepavidin. Arrows on the brightfield photomicrograph point to cell-associated yeasts. Note that areas of
Table II. Effect of Anti-receptor Monoclonal Antibodies on Binding of C. neoformans, EC3b, and EiC3b to MO-M $\phi$

\begin{tabular}{lrrrrr}
\hline & & \multicolumn{4}{c}{ \% Inhibition of binding } \\
\cline { 3 - 6 } \multicolumn{1}{c}{ MAb } & Specificity & $\begin{array}{c}\text { Encapsulated } \\
\text { strain }\end{array}$ & $\begin{array}{c}\text { Acapsular } \\
\text { strain }\end{array}$ & EC3b & EiC3b \\
\hline Anti-CR1 & CR1 & $69 \pm 5$ & $67 \pm 7$ & $1 \pm 9$ & $7 \pm 3$ \\
OKM1 & CR3 & $83 \pm 5$ & $42 \pm 4$ & $-5 \pm 12$ & $81 \pm 2$ \\
OKM10 & CR3 & $76 \pm 3$ & $36 \pm 5$ & $-5 \pm 6$ & $77 \pm 3$ \\
OKM10 F(ab) & CR3 & $34 \pm 4$ & $19 \pm 7$ & $-4 \pm 6$ & $15 \pm 8$ \\
Anti-Leu-M5 & CR4 & $83 \pm 5$ & $79 \pm 5$ & $5 \pm 9$ & $-7 \pm 11$ \\
IV.3 & FCRII & $2 \pm 7$ & $0 \pm 6$ & ND & ND \\
7C3 & PMN & $5 \pm 9$ & $3 \pm 5$ & ND & ND \\
& & & & & \\
\hline
\end{tabular}

$\mathrm{MO}-\mathrm{M} \phi$ were treated with the indicated MAb for $30 \mathrm{~min}$ and then challenged for $30 \mathrm{~min}$ with encapsulated or acapsular $C$. neoformans that had been preopsonized in PHS or with EC3b or EiC3b. Concentrations of MAb used for these experiments were at least five times greater than the maximum inhibitory dose. Results are expressed as percent inhibition of binding compared with MO-M $\phi$ not pretreated with antibody and represent the means \pm SEM of 3 to 10 separate triplicate experiments.

anti-CR3 MAb (OKM1 and OKM10) profoundly inhibited binding of EiC3b to MO-M $\phi$.

Immunofluorescence studies were next performed to see whether CR could be localized to the region of the cryptococcal binding site. Under epifluorescent microscopy, intense staining of MO-M $\phi$ incubated with either anti-leu-M5 (Fig. 4) or OKM1 was seen in the immediate vicinity of bound encapsulated and acapsular $C$. neoformans, but was not seen in other areas of the cell. No fluorescence was visualized when similar experiments were performed with anti-CR1, perhaps because by FACS scanning MO-M $\phi$ stained with anti-CR 1 had approximately 10-fold less fluorescent intensity than MO-M $\phi$ stained with either anti-leu-M5 or OKM1 (data not shown).

Divalent cation requirement for binding. Binding of complement-coated particles to CR3 and CR4, but not CR1, has been shown to require the divalent cations $\mathrm{Ca}^{++}$and $\mathrm{Mg}^{++}$ $(16-18,30,31)$. We therefore next examined the $\mathrm{Ca}^{++}$and $\mathrm{Mg}^{++}$requirements for binding serum-opsonized C. neoformans to MO-M $\phi$ (Table III). Omission of $\mathrm{Ca}^{++}$and $\mathrm{Mg}^{++}$from the culture medium resulted in $>80 \%$ inhibition of binding of both cryptococcal strains.

Inhibition of binding by proteolytic enzymes. Trypsin and human neutrophil elastase both have been shown to affect expression and function of $\mathrm{CR}(19,20,32)$. The effect of these proteases on MO-M $\phi$ binding of $C$. neoformans was therefore investigated (Fig. 5, $a$ and $b$ ). MO-M $\phi$ were incubated with trypsin or elastase, washed, and then incubated with preopsonized yeasts for either 5,15 , or $30 \mathrm{~min}$. Both proteases significantly inhibited binding of encapsulated and, to a lesser extent, acapsular $C$. neoformans to MO-M $\phi$. Inhibition of binding was greatest at $5 \mathrm{~min}$, and diminished progressively (although not entirely) over the time period studied. Because these proteases should only cleave the susceptible pool of external receptors

fluorescence in $B$ correspond to the location of yeasts. No fluorescence is visible on the two MO-M $\phi$ that do not contain cellassociated yeasts. 
Table III. Influence of Divalent Cations on Binding of C. neoformans to MO-M

\begin{tabular}{lcc}
\hline & \multicolumn{2}{c}{ \% Inhibition of binding } \\
\cline { 2 - 3 } & $\begin{array}{c}\text { Encapsulated } \\
\text { strain }\end{array}$ & $\begin{array}{c}\text { Acapsular } \\
\text { strain }\end{array}$ \\
\hline No divalent cations & $83 \pm 2$ & $81 \pm 2$ \\
\hline
\end{tabular}

MO-M $\phi$ were challenged for $5 \mathrm{~min}$ with $C$. neoformans preopsonized in PHS. Medium used for the control group was PBS containing 5.5 $\mathrm{mM}$ glucose, $1.3 \mathrm{mM} \mathrm{Ca}^{++}$, and $0.8 \mathrm{mM} \mathrm{Mg}^{++}$. Medium for the experimental group was PBS with glucose only. Data are expressed as percent inhibition of binding compared with the control group and represent the means $\pm S E M$ of five to six separate triplicate experiments.

$(33,34)$, the loss of inhibition over time is likely secondary to replacement with intact receptors from the internal pool. Treatment of MO-M $\phi$ with elastase resulted in $>70 \%$ inhibition of binding of $\mathrm{EC} 3 \mathrm{~b}$ and $\mathrm{EiC} 3 \mathrm{~b}$, whereas treatment with trypsin inhibited EC $3 \mathrm{~b}$ binding by $26 \%$ and EiC $3 \mathrm{~b}$ binding by $94 \%$.

Effect of temperature and inhibitors of capping and actin on cryptococcal binding. Binding (as opposed to internalization) of
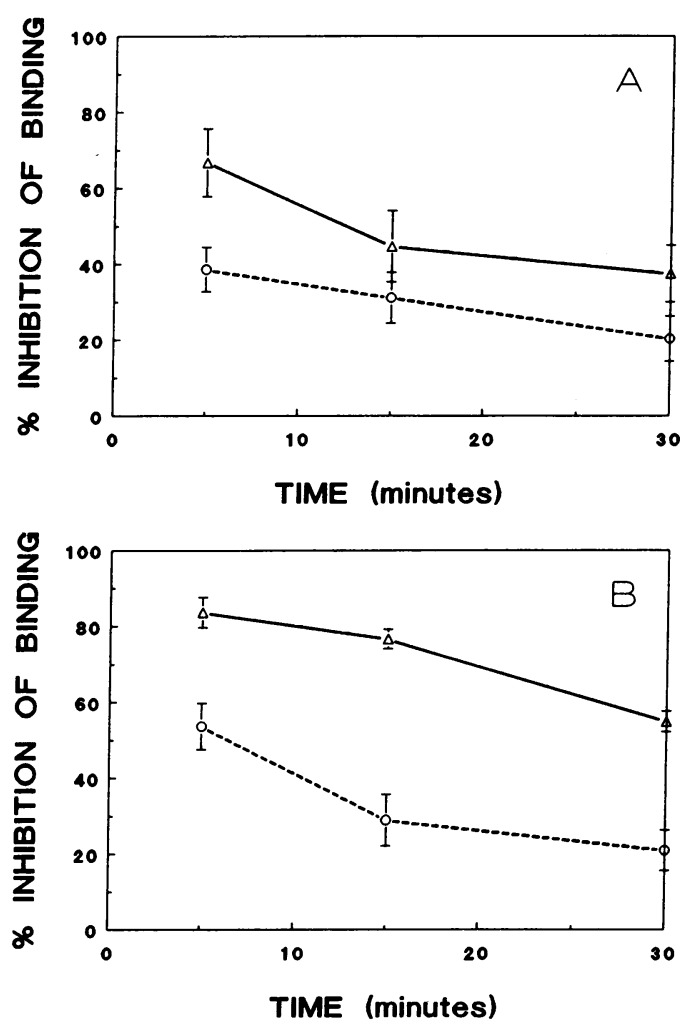

Figure 5. Effect of proteases on binding of $C$. neoformans to MO-M $\phi$. (A) MO-M $\phi$ were trypsin-treated by incubation for $30 \mathrm{~min}$ with 100 $\mu \mathrm{g} / \mathrm{ml}$ trypsin at $4^{\circ} \mathrm{C}$, followed by treatment with $100 \mu \mathrm{g} / \mathrm{ml}$ soy bean trypsin inhibitor and three washes with warm RPMI1640. MO-M $\phi$ were then immediately challenged for 5,15 , or $30 \mathrm{~min}$ with encapsulated $(-\Delta-)$ or acapsular (---O---) strains of $C$. neoformans preopsonized in PHS. Results are expressed as percent inhibition of binding compared with MO-M $\phi$ not treated with protease and represent the means \pm SEM of three triplicate experiments. $(B)$ As in Fig. $3 A$, except MO-M $\phi$ were treated with $10 \mu \mathrm{g} / \mathrm{ml}$ human leukocyte elastase for $60 \mathrm{~min}$ at $37^{\circ} \mathrm{C}$.
Table IV. Effect of Temperature on Binding of C. neoformans to $M O-M \phi$

\begin{tabular}{ccc}
\hline & \multicolumn{2}{c}{ \% Inhibition of binding } \\
\cline { 2 - 3 } Temperature & $\begin{array}{c}\text { Encapsulated } \\
\text { strain }\end{array}$ & $\begin{array}{c}\text { Acapsular } \\
\text { strain }\end{array}$ \\
\hline $4^{\circ} \mathrm{C}$ & $99 \pm 1$ & $26 \pm 2$ \\
\hline
\end{tabular}

MO-M $\phi$ were either chilled to $4^{\circ} \mathrm{C}$ or kept at $37^{\circ} \mathrm{C}$ and then challenged for $5 \mathrm{~min}$ with $C$. neoformans preopsonized in PHS. Data are expressed as percent inhibition of binding compared with the $37^{\circ} \mathrm{C}$ group and represent the means \pm SEM of three separate triplicate experiments.

opsonized erythrocytes to phagocytes does not require energy and occurs at $4^{\circ} \mathrm{C}(18,35)$. We therefore studied whether the situation was similar for $C$. neoformans (Table IV). For these experiments, a 5-min incubation was used. Preliminary experiments established that even at $4^{\circ} \mathrm{C}, \mathrm{MO}-\mathrm{M} \phi$ remained adherent to the cell wells during this incubation period. Nearly no binding of serum-opsonized encapsulated $C$. neoformans to MO-M $\phi$ occurred at $4^{\circ} \mathrm{C}$. In contrast, MO-M $\phi$ bound serumopsonized acapsular yeasts at $4^{\circ} \mathrm{C}$, although binding was somewhat diminished compared with at $37^{\circ} \mathrm{C}$.

The loss of binding of PHS-opsonized encapsulated yeasts at $4^{\circ} \mathrm{C}$ suggested that this binding was an energy-dependent process. Because a population of $\mathrm{CR}$ has been shown to be linked to the cytoskelaton of the cell $(36,37)$, we next examined whether inhibitors of capping and actin filaments would inhibit cryptococcal binding. Both azide and cytochalasin D inhibited binding of PHS-opsonized encapsulated, and to a lesser extent, acapsular yeasts to MO-M $\phi$ (Table V).

\section{Discussion}

The data presented here provide several lines of evidence that, taken together, strongly suggest multiple CR are involved in binding of serum-opsonized $C$. neoformans by MO-M $\phi$. First, monoclonal antibodies directed against CR1, CR3, or CR4 each profoundly inhibited binding of serum-opsonized yeasts to MO-M $\phi$ (Table II). The inhibition was not secondary to nonspecific depression of cellular functions, because these

Table V. Effect of Inhibitors of Capping and Actin Polymerization on Binding of C. neoformans to MO-M $\phi$

\begin{tabular}{lcc}
\hline & \multicolumn{2}{c}{ \% Inhibition of binding } \\
\cline { 2 - 3 } \multicolumn{1}{c}{ Inhibitor } & $\begin{array}{c}\text { Encapsulated } \\
\text { strain }\end{array}$ & Acapsular strain \\
\hline Azide $\left(1.5 \times 10^{-2} \mathrm{M}\right)$ & $53 \pm 5$ & $29 \pm 4$ \\
Cytochalasin $\mathrm{D}\left(8 \times 10^{-5} \mathrm{M}\right)$ & $91 \pm 2$ & $72 \pm 5$ \\
Cytochalasin $\mathrm{D}\left(2 \times 10^{-7} \mathrm{M}\right)$ & $43 \pm 5$ & $30 \pm 5$
\end{tabular}

MO-M $\phi$ were treated with the indicated inhibitor for $30 \mathrm{~min}$ and then challenged for $30 \mathrm{~min}$ with encapsulated or acapsular strains of $C$. neoformans preopsonized in PHS. Results are expressed as percent inhibition of binding compared with MO-M $\phi$ not pretreated with inhibitors and represent the means \pm SEM of three to four separate triplicate experiments. 
MAb did not inhibit binding of organisms opsonized with anticapsular Ig rather than PHS. Moreover, the MAb were specific in their effects on binding of EC3b and EiC3b. Second, immunofluorescent studies localized CR3 and CR4 preferentially to the area of the cryptococcal binding site (Fig. 4). Finally, profound inhibition of binding occurred under conditions where divalent cations were omitted from the system (Table III). Divalent cations have been shown to be necessary for the binding of complement-coated particles by CR 3 and CR4, but not CR 1 $(17,18,30,31)$.

Cleavage of receptors with the proteases trypsin and human neutrophil elastase profoundly inhibited binding of encapsulated, and, to a lesser extent, acapsular $C$. neoformans to MO$\mathbf{M} \phi$ (Fig. 5). Treatment with either trypsin or human leukocyte elastase has been shown to cleave CR1, but not CR3, on activated human PMN as measured by FACS scanning $(20,32)$. In preliminary studies in our laboratory, we have found that elastase treatment of MO-M $\phi$ reduced anti-CR 1 binding by $>50 \%$ as measured by FACS scanning, while having no effect on binding of OKM10 or anti-leu-M5. In contrast, trypsin treatment failed to significantly inhibit binding of any of the three monoclonals (Levitz, S. M., and R. T. Maziarz, unpublished data). However, results obtained by FACS analysis may not correlate with functional data. For instance, if a protease cleaves the complement binding site or a site necessary for signal transduction, but does not cleave the epitope recognized by the MAb, then the receptor may be functionally inactivated without a decrement in FACS staining. Indeed, in our experiments, binding of EC3b and EiC3b were both inhibited when MO-M $\phi$ were protease-treated. Previous investigators also found inhibition of binding of EiC3b to trypsin-treated MO-M $\phi$ (19).

Involvement of multiple $\mathrm{CR}$ in binding of a complementcoated particle appears not to be limited to $C$. neoformans, as Payne and Horwitz, using conditions similar to ours, demonstrated $>60 \%$ inhibition of MO-M $\phi$ binding of serum-opsonized Legionella pneumophilia by MAb directed against either CR 1 or CR3 (38). In our studies, anti-CR1 and anti-leu-M5 profoundly inhibited binding of $C$. neoformans to MO-M $\phi$ under conditions where these MAb failed to appreciably inhibit binding of EC3b and EiC3b. Thus, these MAb appear to be inhibiting cryptococcal binding by mechanisms distinct from simple physical blockade of complement-binding sites.

Generally, binding is thought to be a passive process in that it does not require energy and will occur in the cold, whereas actual phagocytosis is energy-dependent, requires a minimum temperature of $18^{\circ} \mathrm{C}$, and is accompanied by assembly and cross-linking of actin filaments in the cell's cytoskeleton (35). However, binding of serum-opsonized encapsulated $C$. neoformans to MO-M $\phi$ was profoundly inhibited by cold and by inhibitors of actin assembly and receptor capping (Tables IV and V). The mechanism(s) responsible for the temperature and cytoskeleton requirements of binding remain speculative. One possibility is that the CR on MO-M $\phi$ bind complement components on encapsulated yeasts very weakly. Active migration of multiple CR to the organism, perhaps accompanied by some degree of cytoskeletal reorganization, would then be required to reach the critical strength necessary to hold the organism in place. Interestingly, after receptor-ligand binding, several receptors on phagocytes, including CR 1 and CR3, are recruited to the cytoskeleton and specifically associate with actin filaments $(36,37)$. Moreover, a subset of plasma membrane CR3 has recently been described that appears to be involved in phagocytosis of particles by several different receptors includ- ing $\mathrm{CR} 1$ and FcR. This subset is attached to the cytoskeleton of the phagocyte and its function is inhibited by both cytochalasin and MAb directed at sites on CR3 distinct from the complement-binding site (39). For FMLP receptors, receptor occupancy has been shown to lead to actin polymerization, and, concomitantly, conversion of the receptor complex from a fast dissociating to a slow dissociating form $(40,41)$. Thus, cytoskeletal changes could contribute to cryptococcal binding by increasing the affinity of CR for ligands on the yeast's surface. Recently, both attachment and phagocytosis of unopsonized Histoplasma capsulatum (but not complement-coated erythrocytes) to human MO-M $\phi$ was shown to be inhibited by cytochalasin D (23).

The requirements for multiple $C R$, energy, and actin may help explain why binding of serum-opsonized encapsulated $C$. neoformans is so inefficient, despite the dense accumulation of iC3b on the surface of the organism $(8,9,11)$. However, it still remains unclear what factors on the organism contribute to this inefficient binding. For complement-coated erythrocytes, clustering of $\mathrm{C} 3$ fragments has been shown to lead to more efficient binding by phagocytes than when the $\mathrm{C} 3$ molecules are randomly distributed on the surface (42). It is thus possible that the distribution of $\mathrm{C} 3$ fragments on the capsular surface thwarts efficient phagocytic binding. Alternatively, the site on the $\mathrm{C} 3$ molecule that interacts with the ligand-binding site on phagocytic CR may not be optimally exposed so that high-affinity binding can occur. Further studies on the molecular biology of complement activation and deposition on cryptococcal capsule are needed to answer these questions.

After binding of a particle to phagocytic receptor(s), internalization of the particle may or may not take place, depending upon the particular receptor(s) involved and its state of activation $(24,43)$. Whereas FCR and mannose receptors constitutively phagocytose bound particles, $C R$ do not, although under certain conditions they can be upregulated to do so (44). Moreover, CR may collaborate with internalization-promoting receptors to phagocytose a particle. For example, mannose receptors can synergize with CR to efficiently internalize complement-coated zymosan or Leishmania donovani promastigotes and amastigotes $(35,44,45)$. Consistent with these observations, of the serum-opsonized encapsulated $C$. neoformans bound to MO-M $\phi$, only $23 \%$ actually was internalized (Table I). In contrast, 64 and $88 \%$ of bound Ig-opsonized encapsulated and serum-opsonized acapsular $C$. neoformans, respectively, were internalized. The ability of MO-M $\phi$ to phagocytose serum-opsonized acapsular yeasts likely relates to exposed mannans, $\beta$-glucans or other ligands exposed on the cell wall of the organism. Such cell wall ligands are masked from the surface of encapsulated organisms by capsule and thus are not available to bind to their appropriate receptor. These phagocytosis data are consistent with results previously reported using mouse peritoneal macrophages $(5,29)$.

Thus, our data provide evidence that binding of serum-opsonized encapsulated $C$. neoformans by MO-M $\phi$ occurs by a novel mechanism. First, multiple CR are involved. Second, binding is an energy-dependent event that does not trigger phagocytosis. The disparities between our results with complement-opsonized $C$. neoformans, and those reported in the literature using other complement-opsonized particles, emphasize the vast repertoire of responses the macrophage has at its disposal. Further characterization of the receptors involved in recognition of $C$. neoformans, and the biochemical events triggered after receptor binding, should provide important insights 
into the pathogenesis of cryptococcosis as well as the mechanisms of phagocytic function.

\section{Acknowledgments}

The authors thank Drs. Simon Newman, John Bennett, Patricia Roa, David Melnick, and Michael Fanger for generous gifts of reagents, and Dr. Richard Diamond for helpful discussions and critically reading this manuscript.

This work was supported in part by grants to Dr. Levitz from the National Institutes of Health (AI-00658 and AI-25780). Dr. Levitz is a recipient of a Physician-Scientist Award from the National Institutes of Health.

\section{References}

1. Diamond, R. D. 1989. Cryptococcus neoformans. In Principles and Practice of Infectious Diseases. 3rd ed. G. L. Mandell, R. G. Douglas, and J. E. Bennett, editors. Churchill Livingstone, New York. 1980-1989.

2. Gentry, L. O., and J. S. Remington. 1971. Resistance against Cryptococcus conferred by intracellular bacteria and protozoa. J. Infect. Dis. 123:22-31.

3. Monga, D. P. 1981. Role of macrophages in resistance of mice to experimental cryptococcosis. Infect. Immun. 32:975-978.

4. Granger, D. L., J. R. Perfect, and D. T. Durack. 1986. Macrophage-mediated fungistasis in vitro: requirements for intracellular and extracellular cytotoxicity. J. Immunol. 136:672-680.

5. Levitz, S. M., and D. J. DiBenedetto. 1988. Differential stimulation of murine resident peritoneal cells by selectively opsonized encapsulated and acapsular Cryptococcus neoformans. Infect. Immun. 56:2544-2551.

6. Levitz, S. M., and D. J. DiBenedetto. 1989. Paradoxical role of capsule in murine bronchoalveolar macrophage-mediated killing of Cryptococcus neoformans. J. Immunol. 142:659-665.

7. Diamond, R. D., J. E. May, M. A. Kane, M. M. Frank, and J. E. Bennett. 1974. The role of the classical and alternate complement pathways in host defenses against Cryptococcus neoformans infection. J. Immunol. 112:2260-2270.

8. Kozel, T. R., G. S. T. Pfrommer, A. S. Guerlain, B. A. Highison, and G. J. Highison. 1988. Role of the capsule in phagocytosis of Cryptococcus neoformans. Rev. Infect. Dis. 10:S436-S439.

9. Kozel, T. R., B. Highison, and C. J. Stratton. 1984. Localization on encapsulated Cryptococcus neoformans of serum components opsonic for phagocytosis by macrophages and neutrophils. Infect. Immun. 43:574-579.

10. Kozel, T. R., and G. S. T. Pfrommer. 1986. Activation of the complement system by Cryptococcus neoformans leads to binding of $\mathrm{iC} 3 \mathrm{~b}$ to the yeast. Infect. Immun. 52:1-5.

11. Kozel, T. R., M. A. Wilson, G. S. T. Pfrommer, and A. M. Schlageter. 1989. Activation and binding of opsonic fragments of $\mathrm{C} 3$ on encapsulated Cryptococcus neoformans by using an alternative complement pathway reconstituted from six isolated proteins. Infect. Immun. 57:1922-1927.

12. Kozel, T. R., and C. A. Hermerath. 1984. Binding of cryptococcal polysaccharide to Cryptococcus neoformans. Infect. Immun. 43:879-886.

13. Kozel, T. R., and E. C. Gotschlich. 1982. The capsule of Cryptococcus neoformans passively inhibits phagocytosis of the yeast by macrophages. J. Immunol. 129:1675-1680.

14. Kozel, T. R. 1983. Dissociation of a hydrophobic surface from phagocytosis of encapsulated and non-encapsulated Cryptococcus neoformans. Infect. Im mun. 39:1214-1219.

15. Law, S. K. A. 1988. C3 receptors on macrophages. J. Cell Sci. 9:S67-S97.

16. Myones, B. L., J. G. Daizell, N. Hogg, and G. D. Ross. 1988. Neutrophil and monocyte cell surface p150,95 has iC3b-receptor (CR-4) activity resembling CR-3. J. Clin. Invest. 82:640-651.

17. Ross, G. D., S. L. Newman, J. D. Lambris, J. E. Devery-Pocius, J. A. Cain, and P. J. Lachmann. 1983. Generation of three different fragments of bound C3 with purified factor I or serum. II. Location of binding sites in the $\mathrm{C} 3$ fragments for factors B and $\mathrm{H}$, complement receptors, and bovine conglutinin. J. Exp. Med. 158:334-352.

18. Wright, S. D., and M. T. C. Jong. 1986. Adhesion-promoting receptors on human macrophages recognize Escherichia coli by binding to lipopolysaccharide. J. Exp. Med. 164:1876-1888.

19. Speert, D. P. S. D. Wright, S. C. Silverstein, and B. Mah 1988. Functional characterization of macrophage receptors for in vitro phagocytosis of unopsonized Pseudomonas aeruginosa. J. Clin. Invest. 82:872-879.

20. Berger, M., R. U. Sorensen, M. F. Tosi, D. G. Dearborn, and G. Doring. 1989. Complement receptor expression on neutrophils at an inflammatory site, the Pseudomonas-infected lung in cystic fibrosis. J. Clin. Invest. 84:1302-1313.

21. Ross, G. D., J. A. Cain, B. L. Myones, S. L. Newman, and P. J. Lachmann.
1987. Specificity of membrane complement receptor type three (CR-3) for betaglucans. Complement. 4:61-74.

22. Bullock, W. E., and S. D. Wright. 1987. Role of the adherence-promoting receptors, CR3, LFA-1, and p150,95, in binding of Histoplasma capsulatum by human macrophages. J. Exp. Med. 165:195-210.

23. Newman, S. L., C. Bucher, J. Rhodes, and W. E. Bullock. 1990. Phagocytosis of Histoplasma capsulatum yeasts and microconidia by human cultured macrophages and alveolar macrophages. J. Clin. Invest. 85:223-230.

24. Newman, S. L., J. E. Devery-Pocius, G. D. Ross, and P. M. Henson. 1984. Phagocytosis of human monocyte-derived macrophages. Independent function of receptors for $\mathrm{C} 3 \mathrm{~b}\left(\mathrm{CR}_{1}\right)$ and $\mathrm{iC} 3 \mathrm{~b}\left(\mathrm{CR}_{3}\right)$. Complement. 1:213-227.

25. Levitz, S. M., D. J. DiBenedetto, and R. D. Diamond. 1987. A rapid fluorescent assay to distinguish attached from phagocytized yeast particles. $J$. Immunol. Methods. 101:37-42.

26. Wright, S. D., P. E. Rao, W. C. VanVoorhis, L. S. Craigmyle, K. Iida M. A. Talle, E. F. Westberg, G. Goldstein, and S. C. Silverstein. 1983. Identification of the $\mathrm{C} 3 \mathrm{bi}$ receptor of human monocytes and macrophages by using monoclonal antibodies. Proc. Natl. Acad. Sci. USA. 80:5699-5703.

27. Melnick, D. A., W. M. Nauseef, S. D. Markowitz, J. P. Gardner, and H. L. Malech. 1985. Biochemical analysis and subcellular localization of a neutrophilspecific antigen, PMN-7, involved in the respiratory burst. J. Immunol. 134:3346-3355.

28. Graziano, R. F., and M. W. Fanger. 1987. Fc $r$ RI and Fc $\tau$ RII on monocytes and granulocytes are cytotoxic trigger molecules for tumor cells. J. Immunol. 139:3536-3541.

29. Griffin, F. M., Jr. 1981. Roles of macrophage Fc and C3b receptors in phagocytosis of immunologically-coated Cryptococcus neoformans. Proc. Natl. Acad. Sci. USA. 78:3853-3857.

30. Micklem, K. J., and R. B. Sim. 1985. Isolation of complement-fragmentiC3b-binding proteins by affinity chromatography. Biochem. J. 231:233-236.

31. Malhotra, V., N. Hogg and R. B. Sim. 1986. Ligand binding by the p150,95 antigen of U937 monocytic cells: properties in common with complement receptor type 3 (CR3). Eur. J. Immunol. 16:1117-1123.

32. Tosi, M. F., and M. Berger. 1988. Functional differences between the $\mathbf{4 0}$ $\mathrm{kDa}$ and 50 to $70 \mathrm{kDa}$ IgG Fc receptors on human neutrophils revealed by elastase treatment and antireceptor antibodies. J. Immunol. 141:2097-2103.

33. O'Shea, J. J., E. J. Brown, B. E. Seligmann, J. A. Metcalf, M. M. Frank, and J. I. Gallin. 1985. Evidence for distinct intracellular pools of receptors for C3b and C3bi in human neutrophils. J. Immunol. 134:2580-2587.

34. Lacal, P., F. Pulido, F. Sanchez-Madrid, and F. Mollinedo. 1988. Intracellular location of T200 and Mol glycoproteins in human neutrophils. J. Biol. Chem. 263:9946-9951.

35. Silverstein, S. C., S. Greenberg, F. DiVirgilio, and T. H. Steinberg. 1989. Phagocytosis. In Fundamental Immunology. 2nd ed. W. E. Paul, ed. Raven Press Ltd., New York. 703-720.

36. Jack, R. M., R. M. Ezzel, J. Hartwig, and D. T. Fearon. 1986. Differential interaction of the $\mathrm{C} 3 \mathrm{~b} / \mathrm{C} 4 \mathrm{~b}$ receptor and $\mathrm{MHC}$ class I with the cytoskeleton of human neutrophils. J. Immunol. 137:3996-4003.

37. Suchard, S. J., and L. A. Boxer. 1989. Characterization and cytoskeletal association of a major cell surface glycoprotein, GP 140, in human neutrophils. $J$. Clin. Invest. 84:484-492.

38. Payne, N. R., and M. A. Horwitz. 1987. Phagocytosis of Legionella pneumophila is mediated by human monocyte complement receptors. J. Exp. Med. 166:1377-1389.

39. Graham, I. L., H. D. Gresham, and E. J. Brown. 1989. An immobile subset of plasma membrane CD1 1b/CD18 (Mac-1) is involved in phagocytosis of targets recognized by multiple receptors. J. Immunol. 142:2352-2358.

40. Jesaitis, A. J., J. R. Naemura, R. G. Painter, L. A. Sklar, and C. G. Cochrane. 1983. The fate of an $\mathrm{N}$-formylated chemotactic peptide in stimulated human granulocytes. J. Biol. Chem. 258:1968-1977.

41. Jesaitis, A. J., J. O. Tolley, G. M. Bokoch, and R. A. Allen. 1989. Regulation of chemoattractant receptor interaction with transducing proteins by organizational control in the plasma membrane of human neutrophils. J. Cell Biol. 109:2783-2790.

42. Hermanowski-Vosatka, A., P. A. Detmers, O. Gotze, S. C. Silverstein, and S. D. Wright. 1988. Clustering of ligand on the surface of a particle enhances adhesion to receptor-bearing cells. J. Biol. Chem. 263:17822-17827.

43. Bianco, C., F. M. Griffin, Jr. and S. C. Silverstein. 1975. Studies of the macrophage complement receptor. Alteration of receptor function upon macrophage activation. J. Exp. Med. 141:1278-1290.

44. Ezekowitz R. A. B., R. B. Sim, G. G. MacPherson, and S. Gordon. 1985. Interaction of human monocytes, macrophages, and polymorphonuclear leukocytes with zymosan in vitro. Role of type 3 complement receptor and macrophage-derived complement. J. Clin. Invest. 76:2368-2376.

45. Blackwell, J. M., R. A. B. Ezekowitz, M. B. Roberts, J. Y. Channon, R. B Sim, and S. Gordon. 1985. Macrophage complement and lectin-like receptors bind Leishmania in the absence of serum. J. Exp. Med. 162:324-331. 